\title{
Retraction Note to: The IncRNA XIST promotes colorectal cancer cell growth through regulating the miR-497-5p/FOXK1 axis
}

Nan Wang, Jia-Xing He, Guo-Zhan Jia, Ke Wang, Shuai Zhou, Tao Wu* and Xian-Li He*

Retraction to: Cancer Cell Int (2020) 20:553 https://doi.org/10.1186/s12935-020-01647-4

The Editors-in-Chief have retracted this article. Concerns were raised regarding Figure 2, specifically:

- Figure 2c: part of the top right panel appears to be partly covered by a white circle.

- Figure 2d: the si-NC panels appear to partially overlap.

- Figure 2e: the si-XIST HT29 panel appear to partially overlap with the siXIST HT29 panel and the si-XIST SW480 panel of Figure 2d.
The Editors-in-Chief therefore no longer have confidence in the reliability of the data reported in the article.

Xian-Li He has stated on behalf of all authors that the authors agree with this retraction.

Published online: 14 September 2021

\section{Publisher's Note}

Springer Nature remains neutral with regard to jurisdictional claims in published maps and institutional affiliations. 